\title{
PARALYTIC DEFORMITY IN THE LOWER LIMB
}

\author{
W. J. W. Sharrard, Sheffield, England \\ From the Department of Orthopaedics, University of Sheffield, and the Children's Hospital, Sheffield
}

\begin{abstract}
Because tuberculosis and rickets have become uncommon diseases motor paralysis is now the commonest cause of serious deformity in childhood in Great Britain. The three conditions that share responsibility for this are poliomyelitis, cerebral palsy and spina bifida. The object of this paper is to review the mechanisms of paralytic deformity in these three conditions and to put forward the view that a common etiology underlies the development of paralytic deformity and that its prevention and correction can be achieved by conservative and surgical treatment based on common principles of management.
\end{abstract}

\section{THE PROBLEM}

There is general agreement that paralysis of upper or lower motor neurone may result in deformity, but little account in the literature to explain how or why it may develop. Deformity in association with poliomyelitis has been attributed to oedema in a paralysed limb, shortening of active unopposed muscles, fibrosis in paralysed muscles, adhesions between muscles and their sheaths, stretching of paralysed muscles, failure to stretch paralysed muscles, injudicious splintage, failure to splint, muscle imbalance, posture, gravity and collagen deposition of unknown cause (Jones 1911, Lovett 1922, Ghormley and Allen 1949, Bennett 1952, Seddon 1954).

Deformity in cerebral palsy has often been assumed to be due to spasticity. An analysis of the relationship between spasticity in the triceps surae and the development of equinus deformity showed that relative weakness of voluntary dorsiflexor power was more closely related to the development of equinus than spasticity in the calf (Sharrard 1964a), and other authors have made similar observations (Green and McDermott 1942, Phelps 1957, Pollock 1962).

Deformities in children with open myelomeningocoele, even when present at birth, are probably paralytic in nature but the way in which they develop is not yet understood (Sharrard 1962, 1964b).

\section{TYPES OF DEFORMITY}

Deformity associated with paralysis always starts in the soft tissues. Three mechanisms can produce modifications in length or elasticity of soft tissues.

Acute contracture-In acute neurological disease, such as the acute stage of poliomyelitis, loss of elasticity and shortening may develop in tendons, fasciae, intermuscular septa and joint capsules and ligaments in which collagen is deposited. The changes occur too soon and too rapidly to be caused by disuse or adaptation, and their distribution is unrelated to the paralysis. Similar changes may be found in meningitis, in acute episodes of disseminated sclerosis or after a cerebral vascular catastrophy. Once the acute meningitic stage has subsided the tendency to contracture ceases.

If length can be maintained by stretching the soft tissues during the acute phase of the disease and for a few weeks after it, deformity can be prevented. If passive movements cannot be maintained, as used to occur when tank respirators were employed for respiratory paralysis in poliomyelitis, the deposited collagen becomes converted into strong fibrous tissue and fixed deformity results. 
Postural contracture-If a flail limb is immobilised, or if passive movements are not maintained in its joints, deformity may develop. If there is also oedema, collagen may be deposited in the oedema fluid and lead to contractures and adhesions. A completely flail lower limb adopts a characteristic position of fixed flexion, abduction and lateral rotation at the hip, fixed flexion at the knee and equinus at the ankle (Fig. 1). The daily

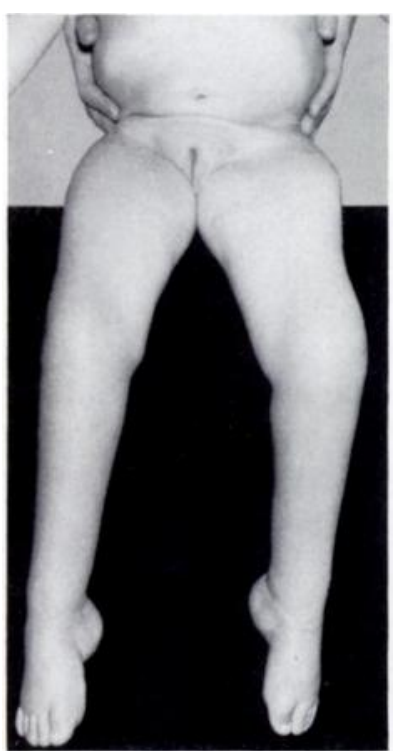

FIG. 1

Postural contractures in completely flail lower limbs. There is fixed flexion of the hips and knees, and severe equinus maintenance of a full range of passive movements combined with measures to lessen oedema is almost always sufficient to prevent the development of deformity at joints over which no muscle is acting.

Deformity from muscle imbalance-This deformity differs in several ways from those already described. It does not occur in any limb with normal muscles; it does not develop in a completely flail limb; and it is seldom seen in adults. It develops slowly and is progressive. The more rapid the rate of growth of the limb the more rapidly does the deformity develop, as was shown in a child who suffered lateral popliteal palsy immediately after birth. He developed 60 degrees of equinus and 45 degrees of varus deformity within six months despite adequate conservative treatment (Figs. 2 and 3).

Although regular passive stretching and splintage may delay its progress, increase in deformity takes place no matter how vigorous the physiotherapy or how firm the splintage. It occurs in all paralytic conditions in children in which there is incomplete paralysis, and its form is related to the altered balance of muscle activity at the joints concerned. A study of the macroscopic and microscopic appearances of muscles and tendons shows differences from the previous contractures. The "short" muscle and tendon has no fibrosis either interstitially or in its sheath unless it has been damaged by over-vigorous stretching. Microscopically, it is often indistinguishable from normal and the only abnormality is that its total length is less than it should be. It is not shorter than it was at the age at which the paralysis developed, but it is short relative to the length of the skeleton. The paretic or paralysed antagonist muscle may show interstitial fibrosis but this does not produce shortening; if there is any fault in this muscle it is that it is too long.

The degree and complexity of deformity that may arise from the isolated pull of one muscle is shown in Figure 4. Two years before the foot had been normal. Acute neuritis of the roots of the cauda equina had resulted in paralysis of all muscles below the knee except the tibialis anterior, which was working almost normally. Apart from the tibialis anterior tendon, which was the shortest structure, the extensor hallucis longus tendon, the dorsal and medial capsules of the ankle and mid-foot joints, the tibialis posterior tendon, both long toe flexor tendons and the tendo calcaneus were also short relative to the bony structures and needed to be divided to obtain correction (Fig. 5). The peroneal muscles, in contrast, were longer than on the normal side, so that, when the deformity was corrected, there was a surplus of tendon length that had to be reefed.

In this example there is a paradoxical situation. The paralysed tibialis posterior, extensor hallucis longus and long toe flexors were too short and the paralysed peronei were too long. It is this situation that has probably produced the confusion of thought about the part played by paralysed muscles in the production of fixed deformity. The paralysed muscles may become too short or too long, depending on the position of the joint over which they no longer have control. The constant factor is the unopposed active muscles; they are always too short and, while growth is still active, their relative shortness increases progressively. 


\section{PATHOGENESIS OF SOFT-TISSUE SHORTENING}

The factors responsible for the rapid deposition of collagen in acute neurological contracture are not known. Whatever they are, they are different from those of postural deformity or deformity from muscle imbalance. There is no relationship to the distribution of the paralysis: the process develops rapidly and there is often muscle tenderness and pain on attempting to stretch the affected structures.

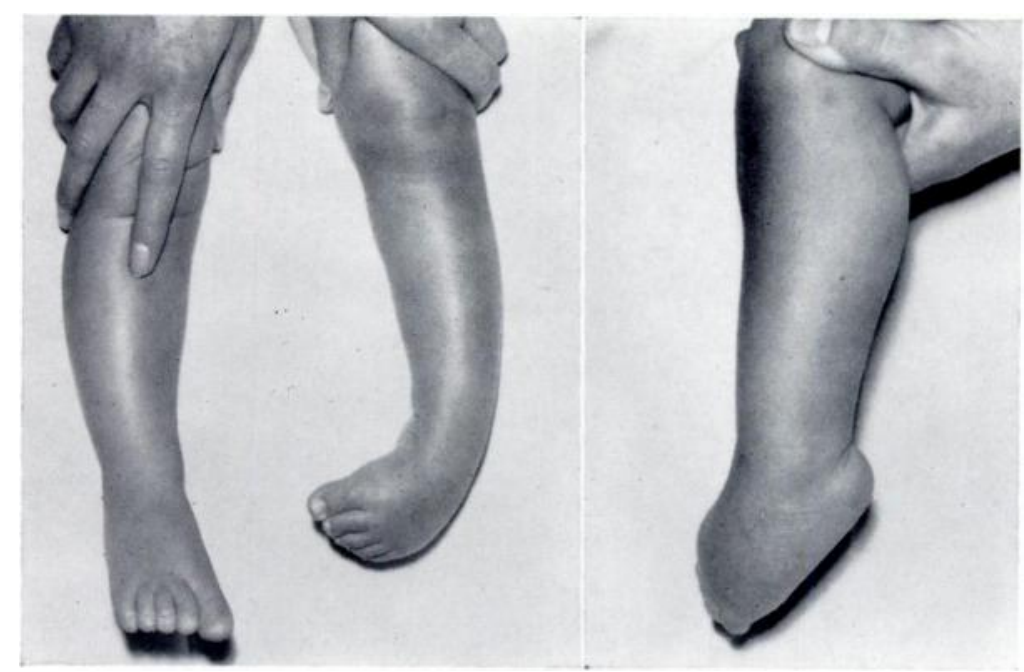

Fig. 2

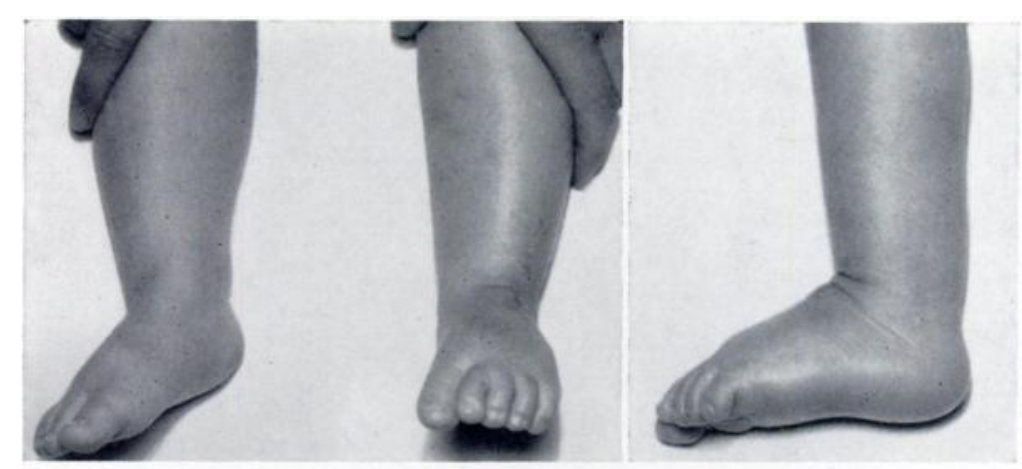

Fig. 3

Figure 2-Paralytic equinovarus deformity of the left foot, caused by lateral popliteal paralysis at birth, at the age of 6 months. The severe deformity was fixed and had occurred despite splints and passive stretching. Figure 3-The same child aged 18 months; correction has been maintained after elongation of the tendo calcaneus and transfer of tibialis posterior to the dorsum of the foot one year previously.

Immobilisation of the foot of a rabbit in dorsiflexion causes shortness of the tibialis anterior (Alder, Crawford and Edwards 1959). The effect is less in adult rabbits than in young rabbits. In growing animals the longitudinal growth of the muscle belly decreased in comparison with the normal, but this did not occur in adult animals (Wiley 1959). These findings correspond to the situation in man. Although continued maintenance of a particular posture, whether imposed by gravity in a flail limb or by unopposed muscle action in a partly paralysed limb, can produce fixed deformity, the effect in childhood is much increased by the diminished growth of muscles on the active side of the deformity. Their opponent muscles 
are either of normal length or, if the deformity becomes severe enough to extend beyond the normal range of movement at the joint concerned, they become longer than normal.

Once deformity has developed, a vicious circle of cause and effect is produced. The stronger muscle is able to act with increasing mechanical advantage and the paretic muscle suffers increasing mechanical disadvantage. Against the combination of posture, diminished muscle

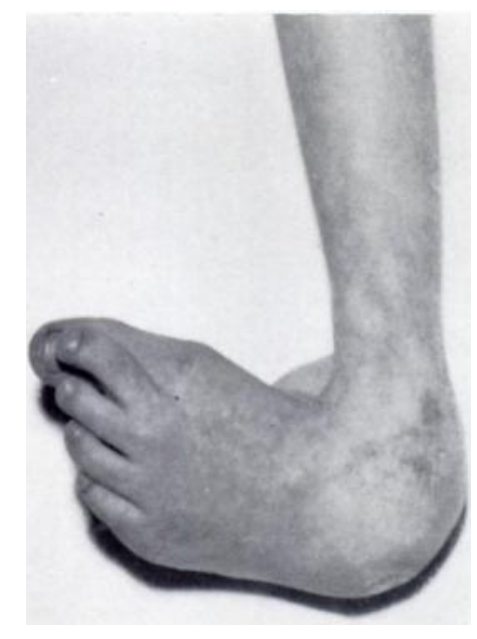

FIG. 4

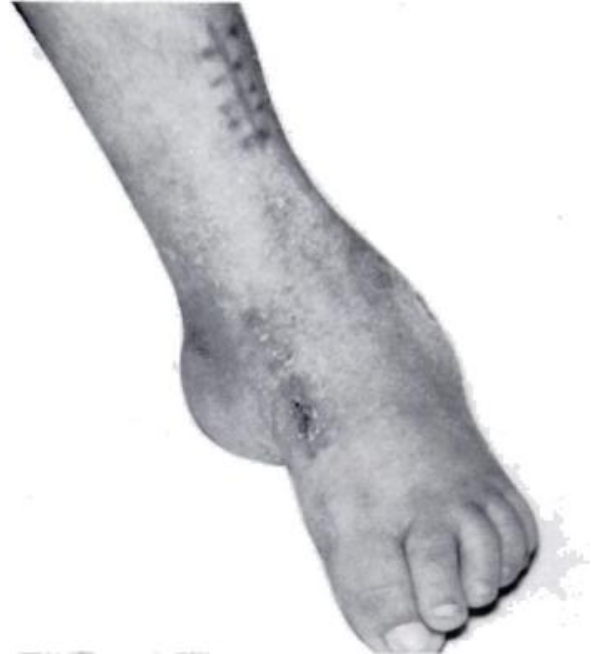

FIG. 5

Figure 4-Calcaneo-varus deformity of the left foot following neuritis of the cauda equina, and caused by the solitary action of the tibialis anterior. Figure 5-The same foot after correction by soft-tissue division and lateral transfer of the tibialis anterior tendon.

growth and increasing muscle imbalance it is no surprise that splintage or short periods of daily physiotherapy fail to prevent the steady progression of deformity. When there is no muscle imbalance, or in an adult in whom the growth factor does not apply, it is more likely that splintage and physiotherapy can succeed in lessening or preventing deformity.

\section{MULTIPLE DEFORMITIES}

The child shown in Figure 6 suffered from acute poliomyelitis during the first six months of life. Eleven years later, when he first attended an orthopaedic department, his left lower limb, which was flail, had acute-stage flexion contracture of the hip, flexion contracture of the knee and equinovarus deformity of the foot. The right lower limb, which was flail below the knee, had a postural equinovalgus deformity. At the right hip, paralysis of all the gluteal muscles in the presence of active hip flexor and adductor muscles had resulted in severe flexion and adduction deformity with dislocation. The trunk showed a combination of deformities from contracture of the lumbo-sacral fascia and scoliosis from imbalance of trunk muscles on the two sides.

The child shown in Figure 7 suffered from cerebral palsy with spastic tetraplegia. In earlier years she had had physiotherapy and by the age of four years was able to stand with support though with her hips and knees flexed. She was also a deaf mute: this had not been recognised by the Education Authorities who assessed her as ineducable and she was in an institution for the next seven years with no treatment of any kind. She developed fixed flexion and adduction of both hips which dislocated; fixed flexion of both knees so gross that the heels touched the buttocks; equinus deformity of both ankles and an extremely severe scoliosis, so that she was unable to sit or lie except on one side. Her intelligence was later found to be normal. 


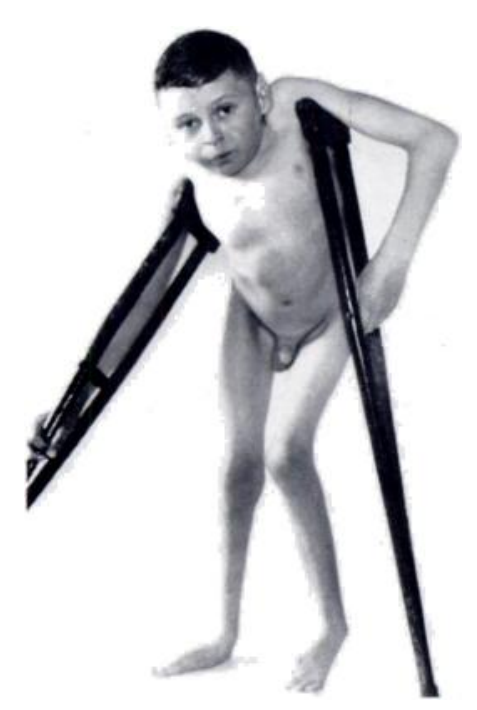

FIG. 6

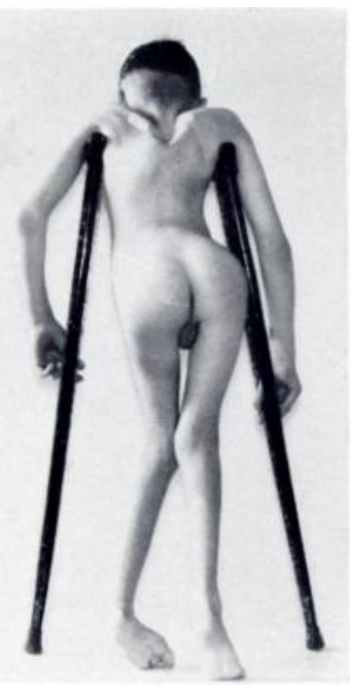
deformities associated with cerebral palsy.
deformities after poliomyelitis. Figure

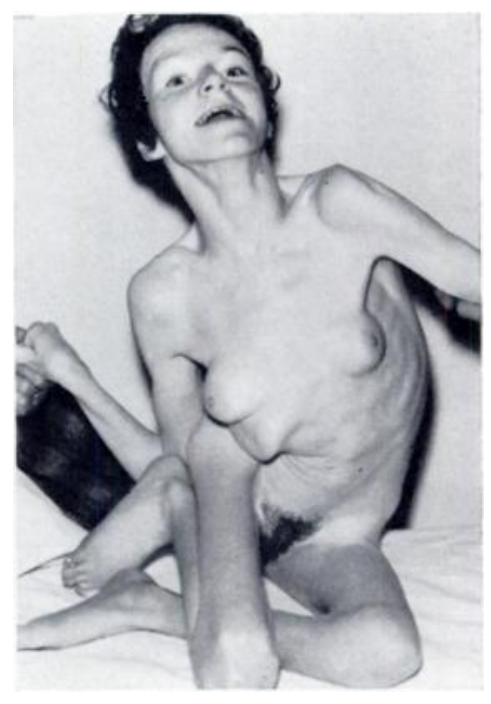

Fig. 7

Figure 6-Untreated multiple paralytic deformities after poliomyelitis. Figure 7-Untreated multiple paralytic

These two patients illustrate what can happen in untreated paralytic deformity. None of the deformities need have been so severe and indeed most of them could have been prevented.

\section{THE LIMITS OF ACCEPTANCE OF DEFORMITY}

Some degree of deformity at a joint may be acceptable when related to the loss of function that has already been dictated by the paralysis. The last 20 degrees of lateral and medial rotation of the hip, of flexion of the knee or of dorsiflexion of the ankle are not needed except in athletic or gymnastic pursuits, of which a child may in any event be incapable. It is unsafe. however, to apply this principle to all deformities. Certain deformities are always likely to be detrimental to function and should be corrected if they exceed certain limits.

At the hip more than 25 degrees of flexion deformity, even if compensated by lumbar lordosis, is not acceptable unless there is extreme paralysis or mental retardation. Progressive limitation of abduction at the hip should always be taken very seriously and, if the range of abduction becomes less than 20 degrees, paralytic dislocation is likely to ensue. Fixed medial rotation or fixed abduction is also unacceptable.

At the knee a few degrees of fixed flexion may be acceptable but, beyond this, the gait kecomes seriously affected and there may develop secondary deformities at the hip and ankle. Recurvatum, valgus or varus of more than a few degrees is also unacceptable in most patients.

At the ankle, limitation of dorsiflexion can usually be accepted provided that the ankle can be dorsiflexed to a little above a right angle. Fixed equinus should be corrected except in a flail lower limb, in which a few degrees of fixed equinus may aid secondary extension of the knee in walking. Some limitation of inversion or eversion of the heel or forefoot is often compatible with satisfactory foot function, but fixed varus deformity of the heel or forefoot is seldom ever acceptable and should te corrected.

\section{ASSESSMENT OF FIXED DEFORMITY}

The examination of a patient with paralytic deformity requires more than an estimate of the loss of range of joint movement. In the early stages of development of a deformity, before changes have occurred in joints, capsules and ligaments, the limitation of movement is determined by the relative shortness of a muscle or a group of muscles. Examination must

VOL. 49 B, NO. 4, NOVEMBER 1967 
be directed to determining which muscles are the most affected. At the hip, for instance, fixed lateral rotation may be due to a short iliopsoas tendon or to tightness of the short lateral rotators. By estimating the range of medial rotation with the hip flexed or extended it is often possible to show that one or other group is primarily involved. Similarly, fixed flexion of the hip that disappears when the hip is abducted suggests that the tensor fasciae latae is primarily involved; if it disappears when the hip is laterally rotated, it is probable that the iliopsoas tendon is mainly affected. Equinus deformity at the ankle that disappears when the knee is flexed indicates primary involvement of the gastrocnemii. Fixed eversion of the forefoot that is relieved when the ankle is plantar-flexed suggests shortness of the peroneus longus, but if it is relieved by dorsiflexion the short muscles are probably the peroneus tertius and extensor digitorum longus.

The assessment of passive movements in the presence of spasticity needs special mention. When muscle spasm is intense it may be difficult to differentiate between shortening of the muscle from over-activity and true relative shortening. In a child slow and steady pressure will usually gain movement of the joint up to a point at which no further range can be obtained because of true muscle shortness. In an adult the power of a muscle group such as the adductors or the triceps surae may be so great that no manual pressure can gain movement. In such circumstances it may be justifiable to use local anaesthesia for temporary relaxation of spasticity or even to give a general anaesthetic to determine the true range of passive movement. In any event, it is always wise, if an operation is to be done upon a spastic patient, to make a final decision as to the degree of fixed deformity when the patient has been anaesthetised; if there is no true fixed deformity surgery is seldom advisable.

\section{ASSESSMENT OF PARALYSIS}

In poliomyelitis there is general agreement that the power of individual muscles should be tested to observe the progress of recovery and to assess the need for tendon transplantation or other measures to prevent deformity (Seddon 1954, Sharrard 1955). It is equally important, and sometimes more difficult, to undertake muscle testing in spina bifida and cerebral palsy. Mortens and Pilcher (1956) emphasised the problems of assessment of muscle power in children below the age of five. In spina bifida there is often need to make some estimate of muscle power in infancy and even during the first few months of life. In cerebral palsy the assessment of voluntary power in muscles antagonistic to strong spastic muscles has proved to be more reliable than was expected and it should be possible to assign the strength of movements in terms of the gradings described by the Medical Research Council (1942). In very young children or in spastic children whose cooperation is poor it is not always possible to assign a power to individual muscles in the foot; but the ability to dorsiflex, evert, invert and plantar-flex as a whole can usually be determined with reasonable accuracy.

\section{MANAGEMENT OF PARALYTIC DEFORMITY}

THE PLACE OF PHYSIOTHERAPY

Physiotherapy has an important part to play in the management of paralytic deformity but its limitations should be appreciated. The techniques available are passive stretching, teaching of correct posture, and muscle exercises.

Passive stretching of soft tissues can prevent acute contracture and postural contracture in a flail limb. Deformity can often be prevented if joints are put through a full range of movement twice a day. It is doubtful whether passive stretching really achieves anything in the prevention of a contracture that arises from muscle imbalance. When a deformity is worsening slowly despite adequate passive stretching there is almost certainly muscle imbalance and physiotherapy alone will not succeed. 
The teaching of correct postures in sitting, standing or walking contributes something to the prevention of paralytic deformity. The patient who persistently walks or stands with the hip laterally rotated will develop valgus deformity at the knee and ankle and lateral rotation contracture at the hip. The child with flail lower limbs who is allowed to lie all day with the hips abducted and flexed will develop a flexion-abduction deformity.

Active exercises to weak muscles, though an essential part of the restoration of function in a partly paralysed limb, are probably the least valuable means of preventing paralytic deformity. Attempts to exercise a weaker muscle almost always result in the development of additional strength in its stronger opponent as well, and when a muscle group is very weak strenuous exercises may even aggravate the imbalance (Sharrard 1955). Once muscle imbalance has been redressed by surgical intervention active exercises may then be invaluable in maintaining muscle balance and in retraining a transplanted muscle.

\section{THE PLACE OF SPLINTAGE}

For the purposes of this paper splintage is taken to include all kinds of external devices of metal, plastic or plaster that may be used in the treatment of deformity, sometimes with the help of traction. Much of what has been said of physiotherapy in the management of paralytic deformity applies to splintage. Its greatest value is an acute or postural contracture. During the acute state of poliomyelitis, splints to maintain a neutral position at each joint are essential during periods between sessions of physiotherapy, but once the acute stage is over continuation of rigid splintage is unnecessary and may be harmful by inhibiting muscle recovery.

In limbs that are not flail splints may have serious disadvantages. The weight of a splint is an additional load, and excessive splintage may make it impossible for the child to walk at all. Moreover if there is sensory loss the splints, no matter how carefully made, can initiate pressure sores. Finally, injudicious splintage may result in spurious correction of deformity (Fig. 8). It is doubtful whether splints can ever completely prevent the development of deformity when there is muscle imbalance, and they should be regarded only as an aid until surgical treatment is possible. In treating spastic paralysis of the lower limbs in cerebral palsy the author has entirely abandoned calipers and splints and uses only physiotherapy with early corrective surgery. In consequence, the problems of deformity far from increasing have lessened, and function in patients uncluttered by extensive apparatus has greatly

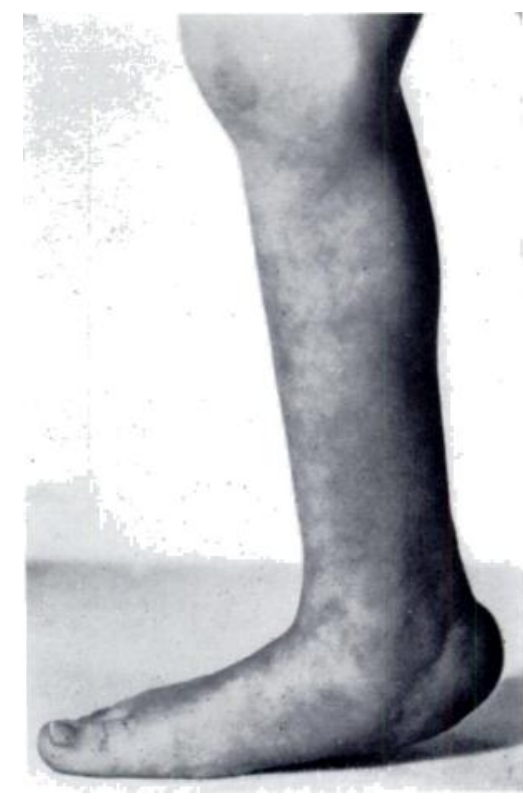

FiG. 8

Spurious correction of equinus deformity. The heel is still in severe equinus and the midtarsal joints have been overstretched by attempts to correct the deformity with
splints. improved. Children with athetoid paralysis may benefit from splintage, but, since muscle imbalance is uncommon in pure athetoid paralysis, fixed deformity does not develop whether splintage is used or not.

The use of traction or serial plasters to correct established paralytic deformity also has considerable limitations. When strong muscles oppose the correction of deformity the force needed to combat them and to stretch strong tendons may be so great as to cause avascular necrosis of the femoral head, epiphysial separation, damage to main vessels, injury to nerves and, in insensitive limbs, serious pressure sores. It is kinder to the patient and to his tissues to achieve correction by surgical division or elongation of the shortened soft tissues.

VOL. 49 B, NO. 4, NOVEMBER 1967 


\section{SURGICAL CORRECTION OF DEFORMITY}

Surgical treatment is indicated in paralytic deformity whenever conservative measures of physiotherapy and splintage have failed, or will inevitably fail, and always when there is muscle imbalance. The greater the deformity the greater is the need for surgical intervention.

Paralytic deformity in the lower limb can be corrected by division or elongation of tendons or muscles, by division of ligaments and joint capsules or by osteotomy, in that order. If the deformity is treated sufficiently early, and has arisen mainly from muscle imbalance, elongation of muscle or tendon alone is nearly always enough. Only in long-standing and severe deformities may ligament or bone division be needed. Skin incisions should be planned to allow plastic correction of skin defects resulting from the correction.

The aim is to regain a normal or almost normal range of movement at the affected joint. If any deformity is left, the hope that it will correct itself after a period in plaster is seldom fulfilled. Sometimes the division of soft tissues has to be very extensive; only when vital structures such as major nerves or vessels are the tightest structures left should the attempt to obtain correction by soft-tissue division be abandoned in favour of bony operation.

Acute stage contractures-The structures usually involved are the fascia lata, the fasciae and tendons behind the knee, the tendo calcaneus and the fasciae of the sole of the foot. Division of the fascia lata at the iliac crest and below the inguinal ligament (Ober 1936) and of the ilio-tibial band and lateral intermuscular septum in the lowest third of the thigh (Yount 1926) will usually correct flexion and abduction contracture at the hip. In a severe deformity the gluteal muscles, the sartorius and the tendon of rectus femoris may need to be mobilised distally and the anterior capsule of the hip divided.

Division of the posterior fascia, elongation of the hamstring tendons and posterior capsulotomy will usually correct flexion deformity at the knee; if the popliteal vessels and nerves limit full correction at the deformity supracondylar extension osteotomy of the lower end of the femur will allow full extension of the knee without increasing tension on vital structures.

At the ankle, elongation of the tendo calcaneus, posterior capsulotomy of the ankle and, sometimes, division of the inferior tibio-fibular ligament will correct equinus deformity.

Postural deformity at flail joints-In a completely flail limb tenotomy is appropriate. Once deformity has been corrected it should not recur provided that passive movement and splintage are maintained (Figs. 9 to 11); with correct conservative management severe deformity should not develop, and in most postural deformities in flail lower limbs subcutaneous tenotomy of the tendo calcaneus is the only operation that is likely to be needed.

Deformity from muscle imbalance-Deformity from muscle imbalance almost always needs surgical treatment by division or elongation of tendons, tendon sheaths, fasciae, ligaments and, if necessary, joint capsules. As in flail joints, tendons of completely paralysed muscles can be divided and left unsutured, but tendons of active muscles must be either elongated or divided and transplanted. A careful assessment before operation will indicate which tendons are active and which inactive, though electrical stimulation may sometimes be needed to be certain.

Calcaneo-varus deformity in the foot of a patient with spina bifida (Fig. 12) is characteristic of muscle imbalance. The only active muscle was the tibialis anterior. The short structures included the skin on the inner side of the ankle and foot, the tibialis anterior and posterior tendons, the tendo calcaneus, the long flexor tendons of the toes, the medial ligaments and capsule of the ankle and subtalar joints and the medial and plantar fasciae of the foot. A $\mathrm{V}$-shaped skin incision, to be sutured as a $\mathrm{Y}$ at the end of the operation, will give access to all the tight structures (Fig. 13). All tendons except the tibialis anterior may be tenotomised freely because they are functionless (Fig. 14); the tibialis anterior tendon is transplanted laterally (Fig. 15). Finally, the medial and posterior ligaments and joint capsules of the ankle and subtalar joints are divided to give a plantigrade foot (Fig. 16). Had the deformity not been 


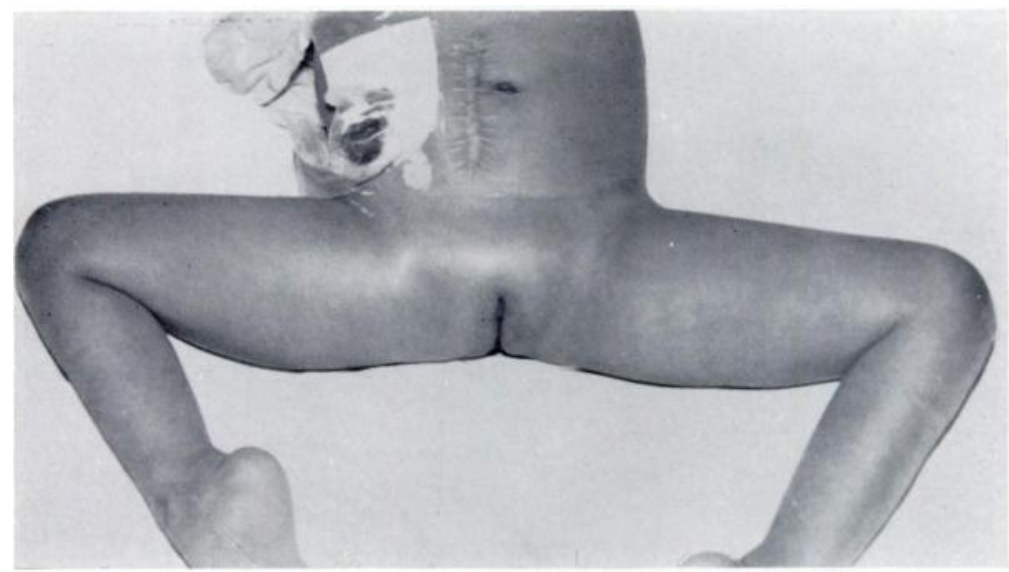

FIG. 9

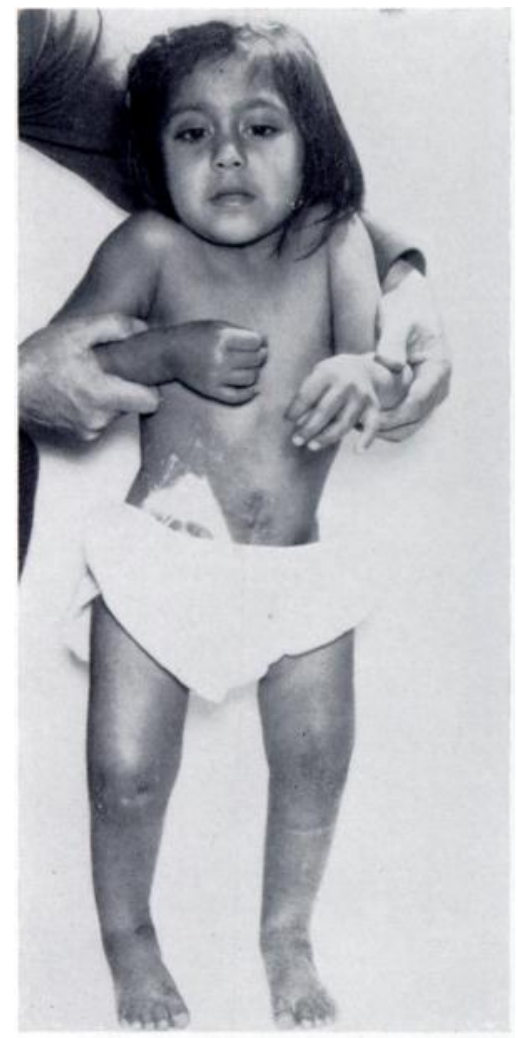

Fig. 10

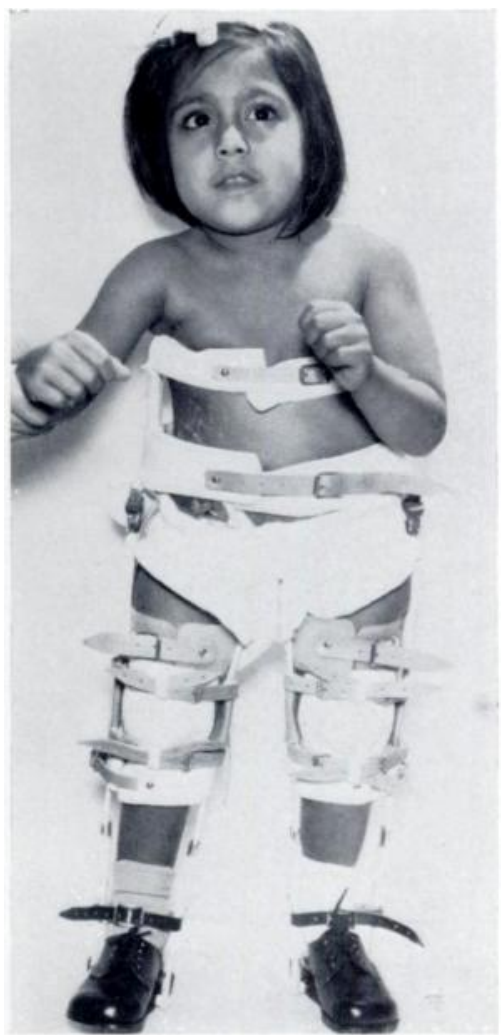

Fig. 11

Figure 9-A child with severe postural deformity. The flail lower limbs have had the deformities aggravated by splintage in abduction for subluxation of the hips. Figure 10-The same child after correction by multiple tenotomies and intertrochanteric osteotomies. Figure 11 -The child walking with calipers with semimobile double hip hinges. 
able to be corrected completely in this way, calcaneal osteotomy (Dwyer 1959) or resection of the calcaneo-cuboid joint (Evans 1961) would have been necessary.

Restoration of muscle balance-The balance of muscle forces acting on a joint may be altered by diminishing the strength of the muscles causing the deformity or by enhancing the strength of their antagonists, or both.

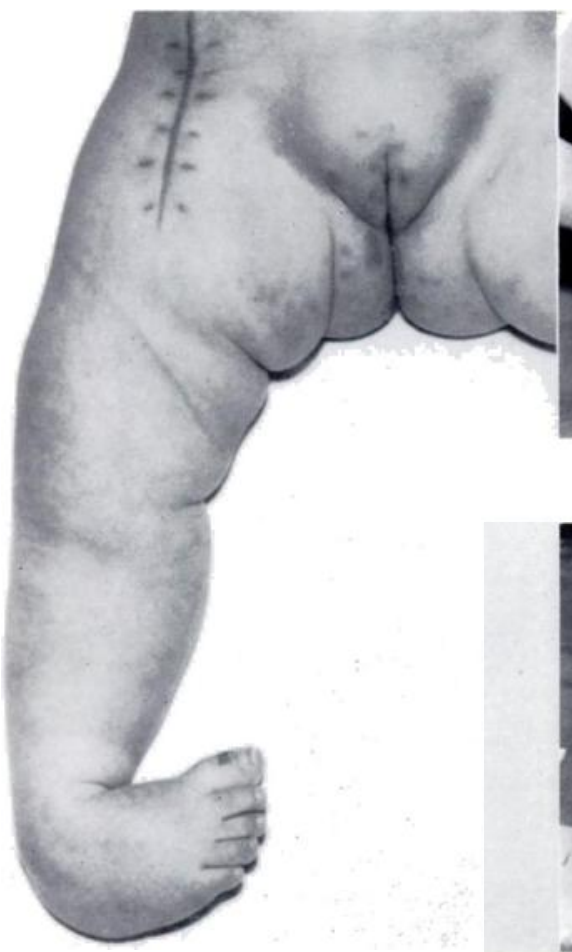

FIG. 12

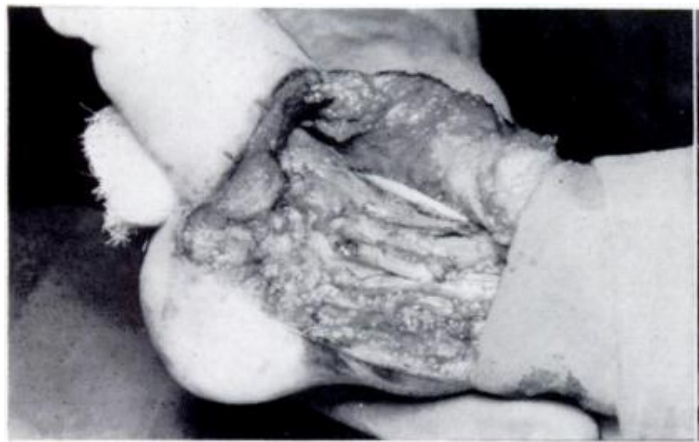

FIG. 15

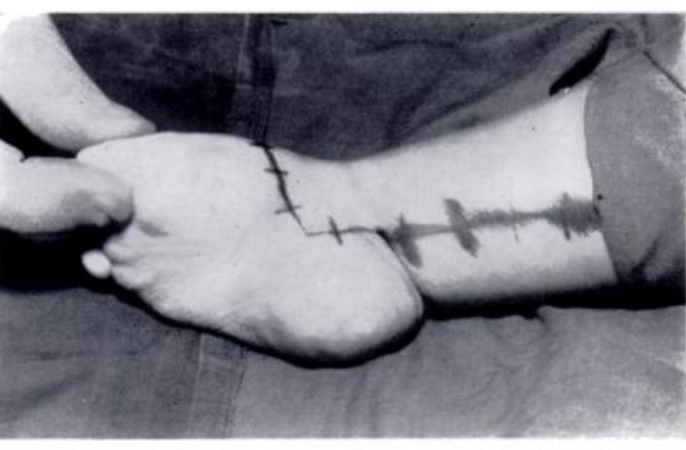

FIG. 13

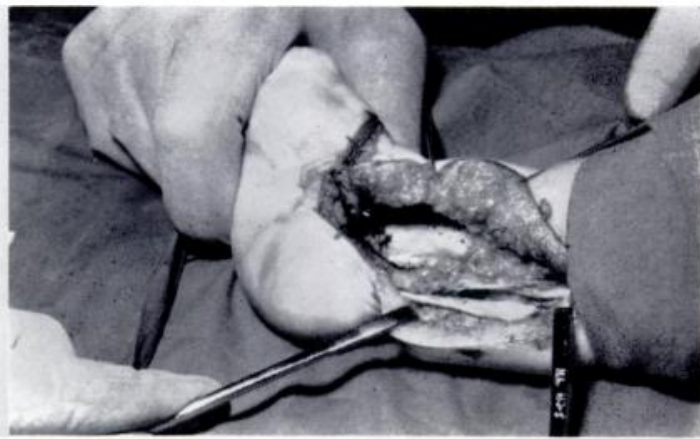

FIG. 14

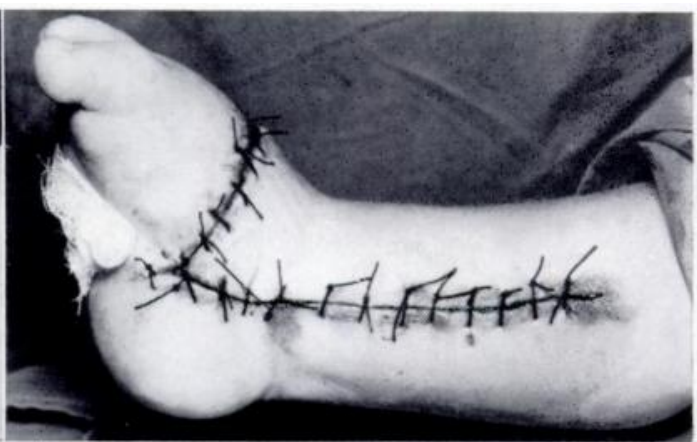

FIG. 16

Figure 12-A child with spina bifida and a paralytic calcaneo-varus deformity. Figure 13-To show the stages of the correction in the same.child. Line of skin incision. Figure 14-Dissection and division of short tendons, fasciae and ligaments. Figure 15-Lateral transfer of tibialis anterior tendon. Figure 16-The ' $Y$ ' skin closure.

Tenotomy, myotomy or tendon elongation in itself diminishes the power of a muscle by approximately one Medical Research Council grade. Strong muscles may be sufficiently weakened by division or elongation to restore muscle balance. Elongation of the tendo calcaneus alone is often sufficient to correct equinus deformity and to remedy muscle imbalance of mild or moderate degree in spastic children. Excessive elongation of the tendo calcaneus 
in the presence of moderately strong ankle dorsiflexors may reduce the power of plantar-flexion of the ankle too much, so that calcaneus deformity may develop later. If dorsiflexion has been graded at three or more, the elongated tendo calcaneus should be sutured with the ankle held at a right angle but not dorsiflexed more than this.

Partial neurectomy is a useful method of diminishing muscle power, particularly in spastic paralysis. Neurectomy was first advocated by Stoffel (1913) but it fell into disrepute because of failure to recognise that fixed deformity cannot be relieved by neurectomy alone, and because of the irrevocable effects of over-extensive muscle denervation in the presence of unsuspectedly strong antagonist muscles (Pollock and Sharrard 1958). Neurectomy after correction of deformity is a very satisfactory procedure provided that the muscle imbalance has been carefully assessed before operation and the amount of nerve division needed has been calculated in advance. Progressive adduction deformity of the hip caused by strong spastic adductors opposed by gluteal muscles of approximately Medical Research Council grade 3 is a common situation in spastic paralysis. It can be corrected by a combination of adductor tenotomy and division of the anterior branch of the obturator nerve (Mathews, Jones and Sperling 1953; Pollock and Sharrard 1958). Total obturator neurectomy is almost never advisable in spastic paralysis because of the danger of secondary fixed abduction deformity. Division of some of the nerves to the gastrocnemius or of the upper nerve supply to the soleus muscle is often a useful adjunct to correction of equinus deformity by gastrocnemius slide operation (Strayer 1950, Pollock 1953). Division of the upper branch of the sciatic nerve to the hamstring muscles is a useful measure in association with proximal elongation of the hamstring origin from the ischial tuberosity when there is marked limitation of straight-leg raising in spastic paralysis.

Tendon transference-Transference of a strong tendon to an alternative site is the most important procedure available for the correction of muscle imbalance in paralytic deformity. Earlier writings on tendon transfer tended to emphasise the new action of the transferred muscle. In the management of paralytic deformity in the lower limb as much importance attaches to the diminution of a deforming force as to the action that a muscle may have when transferred elsewhere.

Not infrequently the transfer of a muscle may require of it an action opposite to or out of phase with that which it previously had (Fig. 3). Several investigations using electromyography have shown that muscles vary in their ability to alter their phase of action after transplantation: but whether or not there is change in phasic action this does not detract from the need to transplant a muscle in a child with progressive deformity, nor does it prevent it from helping to minimise liability to recurrence of deformity.

No better proof can be offered than by the example of hemitransplantation of the tendo calcaneus. In poliomyelitis and in spina bifida it sometimes happens that the only active muscle below the knee is the triceps surae. In these circumstances recurrent severe equinus deformity develops in spite of repeated elongation of the tendo calcaneus. In the absence of any other tendons available for transfer to the dorsum of the foot, it is possible to split the triceps surae in the sagittal plane into two halves and to transfer the lateral half to the dorsum of the foot round the lateral side of the fibula or through the interosseous membrane. This operation has been performed on six occasions and there has been no recurrence of equinus deformity in any patient in a follow-up of four to ten years. In four patients it has been possible to discard all splintage below the knee, an unexpected benefit. Isolated dorsiflexor or plantar-flexor action in either of the remaining parts of the muscle is very weak but there does appear to be some active contraction in the course of walking as a form of muscular tenodesis. The most significant result of this procedure has been its ability to prevent recurrence of deformity by making a muscle act against itself.

The requirements to be met in order to achieve good functional results in tendon transplantation have been well summarised by Barr (1949). When possible the tendon should

VOL. $49 \mathrm{~B}$, NO. 4, NOVEMBER 1967 
be transferred to act in direct line from origin to new insertion, to pass subcutaneously, and to be attached through drill holes in bone under adequate tension. More important than any of these considerations is that the correct tendon should be transferred to the right place. No absolute rules can be offered for any particular situation but, in general, if the shortest and strongest tendon or tendons are transferred to a new position in which they cannot reproduce the deformity that has developed, and preferably to perform a new action that will improve function, the results are likely to be satisfactory. Sometimes, as Mortens and Pilcher (1956) pointed out, tendons transferred to prevent deformities of the foot may restore balance in one direction while destroying it in another, and it may be impossible to achieve both balance and stability by tendon transplantation alone. In these circumstances it is often wise to concentrate on obtaining a balance between dorsiflexion and plantar-flexion

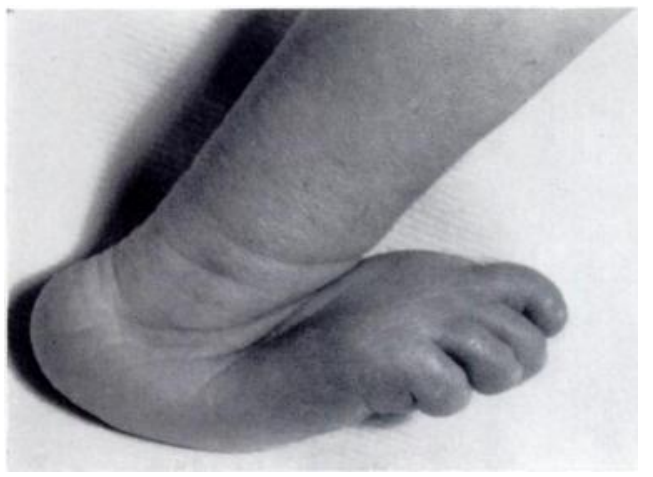

FIG. 17

Congenital paralytic calcaneo-valgus with vertical talus caused by paralysis of the second and third sacral roots. and to counter the valgus effect of gravity on the heel by extra-articular subtalar arthrodesis (Grice 1952).

In some patients muscle imbalance may produce bizarre deformities such as that shown in Figure 17. There is a calcaneo-valgus deformity of the forefoot, slight equinus of the hindfoot and dislocation of the midfoot resulting in a paralytic vertical talus. This deformity arose from the paralysis of the muscles supplied by the second and third sacral segments. The intrinsic muscles of the foot were paralysed and the long toe flexors were weak. A complex state of imbalance at the joints of the ankle and foot was produced. The

dorsiflexors of the ankle, foot and toes, all of which are attached distally in the foot, were acting normally and unopposed by any muscles capable of plantar-flexing the forefoot. The forefoot dislocated into calcaneus relative to the hindfoot which was plantar-flexed by active calf, tibialis posterior and peroneus brevis muscles.

As in non-paralytic congenital vertical talus, correction of the deformity requires elongation of all the dorsiflexor tendons and the dorsal and lateral capsules of the midtarsal joints to allow the forefoot to be reduced on the hindfoot. The midtarsal joint can be stabilised by internal fixation with pins, and the splayed insertion of the tibialis posterior is repaired. The equinus of the hindfoot is corrected by elongation of the tendo calcaneus. There still remains the problem of tendon transfer, or the deformity will recur. Transfer of the tibialis anterior tendon to the neck of the talus, of peroneus tertius to the calcaneus, and peroneus brevis to the tendon of tibialis posterior, produces a balance of action at the ankle and hindfoot and leaves the forefoot to be acted on by the remaining toe flexors and extensors. Sometimes claw deformity of the toes may require transfer of the long toe flexor tendons to the extensor surface of each toe. The multiplicity of these tendon transfer operations has to be faced in this deformity to achieve a stable undeformed foot capable of bearing weight on the sole of a foot that is partly anaesthetic.

Sometimes a tendon transfer may give rise later to another deformity or even to the opposite deformity. Should this happen, it should not necessarily be judged as a failure of the original operation. Conditions may change with growth, and there is no reason why a tendon that later proves to be incorrectly sited should not be rerouted. Whereas neurectomy, once done, is irrevocable, tendon transfer is often capable of further modification if the need arises.

Provided that a child's general condition allows it and that the operation is feasible, there is no lower age limit for the correction of a deformity that is producing disability. In 
children with myelomeningocoele and myelodysplasia with paralytic deformities at birth it may not be opportune to proceed with orthopaedic operations until the child is established and viable. It may be possible to reduce dislocation of the hip by adductor and lateral rotator tenotomy at the third month of life, but posterior iliopsoas transfer may have to be deferred until the child is more than six months old for purely technical reasons of size (Sharrard 1964b). Correction of severe paralytic birth deformities of the foot may have to wait until the foot is large enough to make an operation safe, but it is not correct, as has been suggested by some authors, to wait until the child is five years old or he may have to walk on a deformed foot and so develop serious pressure sores. No paralytic deformity is likely to get better spontaneously; it is very likely to become much worse. Some surgeons have believed that deformity, for instance in the foot, should not be treated surgically until the child is fully grown. They then correct the deformity by extensive bone resection and triple arthrodesis. There is, in my view, no justification for this policy. Deformity, if detected sufficiently early, and provided it has not presented at birth, can almost always be corrected by release of the soft tissues alone and recurrence prevented by appropriate redress of muscle imbalance. There is no interference with growth of the bone in the feet other than that imposed by the paralysis itself, and arthrodesis may, in the ultimate event, never be needed.

Tenodesis needs a final word because it is an operation that has undeservedly fallen into disrepute. Drop foot in a growing child can be corrected by attaching the tendons of the paralysed tibialis anterior and peroneus tertius through a drill hole in the lower half of the tibia. If there are any active ankle plantar-flexors, the correction is only temporary, the tendons gradually elongate and the drop foot recurs. The tenodesed tendons were always said to have stretched but it is more likely that they elongate by growth as a result of the stimulus of active plantar-flexor muscles. If there are no active plantar-flexors-that is, the limb is flail below the knee-tenodesis can give a long-lasting and satisfactory result and may allow heavy splintage to be discarded.

\section{RESULTS}

It is beyond the scope of this article to review in detail the results of management of all varieties of paralytic deformity. Approximately 1,400 patients suffering either from poliomyelitis, cerebral palsy or spina bifida have been treated in the author's care during the past ten years. Only 310 of these have completed growth in their lower limbs but it is satisfactory to note that when it has been possible to follow the course of management outlined in this article, and if the patient was treated before deformity had become excessive. only five have residual lower limb deformity outside the limits of acceptance. This is not meant to infer that all the patients necessarily had good function. Some patients with poliomyelitis were still severely disabled because of extensive paralysis. A few patients with cerebral palsy or hydrocephalus are mentally retarded and are unable to walk because of their poor state of mental development. Even so, they have been spared the additional liabilities of soreness, disfigurement and pain that may accompany uncorrected deformity in this type of patient. In only two children has a deformity been made worse and in both instances there was a technical error in the performance of an operation and the position was able to be retrieved by further procedures.

Poliomyelitis- -Most of the children who suffered paralytic poliomyelitis during the epidemics of 1949 to 1953 have now reached adult life without lower limb deformity or, if there is a residual deformity, it is not disabling and is cosmetically acceptable.

Cerebral palsy-In some centres specialising in the treatment of cerebral palsy there is a tendency to accept deformity that cannot be prevented by conservative management as inevitable, or the patient is not referred for orthopaedic consideration until the deformity has become gross and almost incorrigible. Before the principles proposed in this article were followed, a review of patients at Sheffield Children's Hospital revealed twenty-eight dislocations

VOL. 49 B, NO. 4, NOVEMBER 1967 

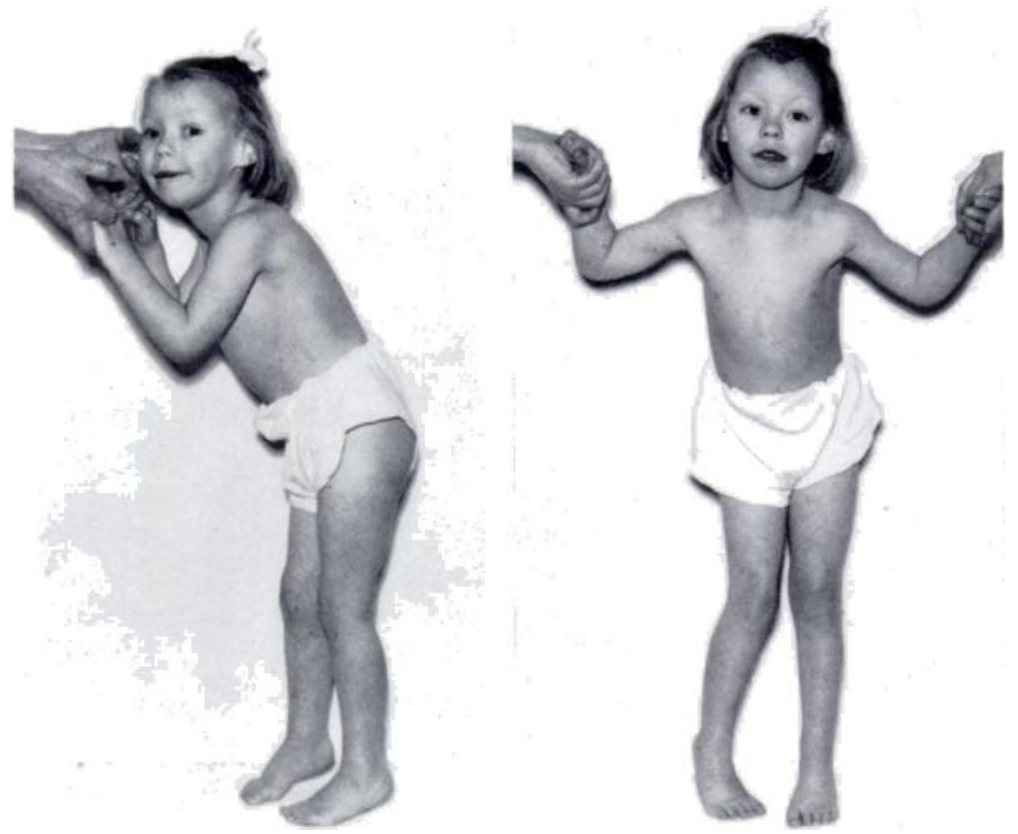

FIG. 18

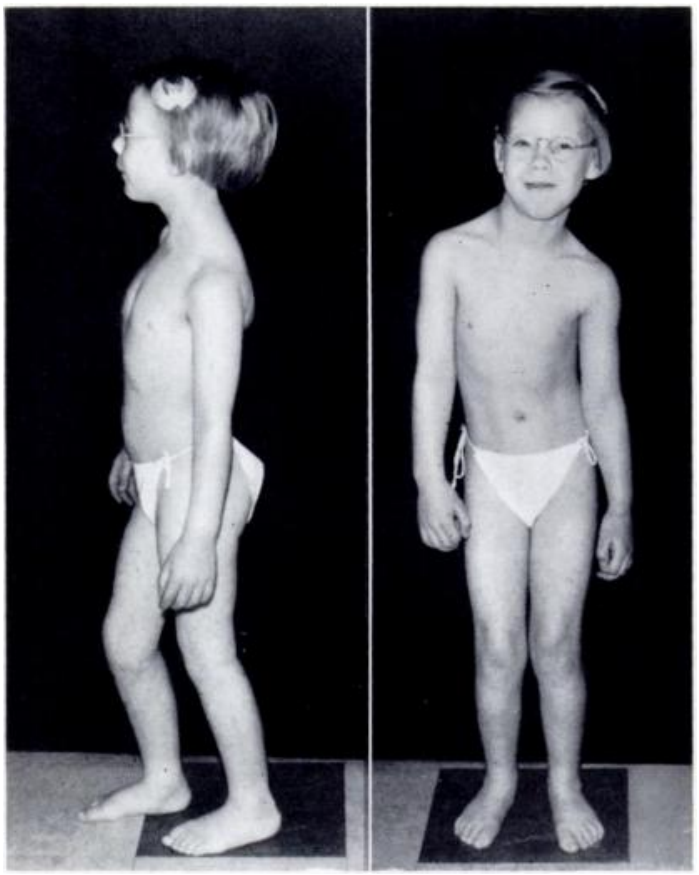

Fig. 19

Figure 18-A spastic child with a paralytic deformity causing flexion and adduction deformity at the right hip and equinus at the right ankle. The knee is flexed but there is no fixed deformity. Figure 19-The same child after correction by bilateral adductor tenotomy, division of the anterior branch of the obturator nerve, iliopsoas tendon lengthening and gastrocnemius slide operations. The child no longer needs support for standing and can walk without appliances. There is a cervico-dorsal scoliosis unrelated to the lower limb deformities. 
or subluxations of the hip in eighty-seven patients (Pollock and Sharrard 1958). Since then, no child has developed dislocation of the hip, serious flexion deformity of the knee or equinus of the ankle, if such deformity was not already present when the child was first referred for treatment. The child shown in Figure 18 was found at initial assessment to have weak gluteal muscles relative to the power of the hip flexors and adductors, and some weakness of dorsiflexion relative to the power of the calf muscles. She was treated conservatively by physiotherapy but, as was expected, she developed flexion and adduction deformity at the hips and equinus deformity at the ankles. Open adductor tenotomy with division of the anterior branch of the obturator nerve and, through the same incision, elongation of the iliopsoas

TABLE I

Orthopaedic Operations in 182 Patients with MENINGOMYELOCOELE FROM 1956 TO 1961

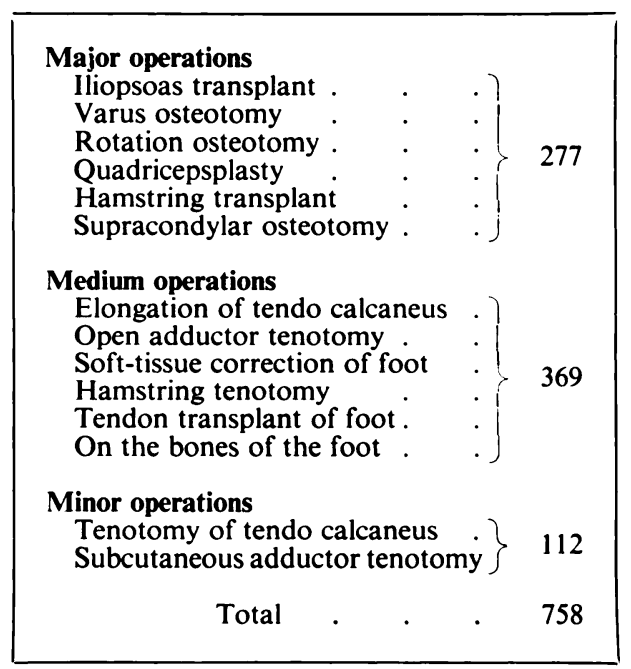

TABLE II

INDEPENDENT WALKING IN MENINGOMYelocoele Survivors over Five Years Old

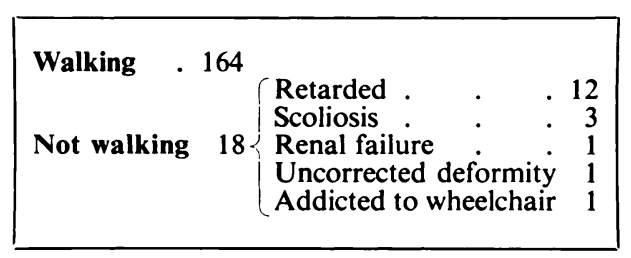

tendon corrected the hip. A gastrocnemius slide operation corrected the equinus deformity of the ankle. The improvement in balance and the complete correction of deformity, which needed no extensive soft-tissue or bony division, is shown in Figure 19. The hips, which had started to subluxate and would have dislocated within the next two years, have remained well reduced.

An interesting result of early correction of ankle and hip deformities in cerebral palsy has been the much lessened liability to knee flexion deformity. Only one-quarter of the number of operations of the type described by Eggers (1952) have been necessary in the last five years compared with the five years preceding that.

Spina bifida-In spina bifida, the results of management of the paralytic hip have already been described (Sharrard 1964b). Corrective operations for deformities of the knee, ankle and

VOL. $49 \mathrm{~B}$, NO. 4, NOVEMBER 1967 
foot present at birth or developing later have needed many different combinations of soft-tissue division, tendon transplantation and osteotomy. Some indication of the number of orthopaedic procedures required in children with meningomyelocoele is shown in Table I. In 182 patients 758 operations have been needed to correct deformity and restore function. That this large number of procedures has been of avail is indicated in Table II. In only eighteen has independent walking not been possible and in only one has this been because of failure to achieve correction of paralytic deformity in the lower limb. Few of the patients with spina bifida are yet old enough to have completed growth in their lower limbs, but it can be said that although the problems and difficulties of management are much greater than in poliomyelitis or cerebral palsy the preliminary results have been no less successful.

\section{SUMMARY}

1. The nature of paralytic deformity arising in poliomyelitis, cerebral palsy and spina bifida is considered and three types of deformity-acute contracture, postural contracture and deformity from muscle imbalance are described.

2. The place of physiotherapy, splintage and surgery in the management of these varieties of paralytic deformity is discussed and the overall results of treatment are reviewed.

\section{REFERENCES}

Alder, A. B., Crawford, G. N. C., and Edwards, R. G. (1959): The Effect of Limitation of Movement on Longitudinal Muscle Growth. Proceedings of the Royal Society, B.150, 554.

Barr, J. S. (1949): The Management of Poliomyelitis: The Late Stage. In Poliomyelitis. Papers and Discussions Presented at the First International Poliomyelitis Conference, p. 201. Philadelphia: J. B. Lippincott Company.

Bennetr, R. L. (1952): Physical Medicine in Poliomyelitis-Points of Emphasis. In Poliomyelitis. Papers and Discussions Presented at the Second International Poliomyelitis Conference, p. 261. Philadelphia: J. B. Lippincott Company.

Dwyer, F. C. (1959): Osteotomy of the Calcaneum for Pes Cavus. Journal of Bone and Joint Surgery, 41-B, 80.

EgGers, G. W. N. (1952): Transplantation of Hamstring Tendons to Femoral Condyles in Order to Improve Hip Extension and to Decrease Knee Flexion in Cerebral Spastic Paralysis. Journal of Bone and Joint Surgery, 34-A, 827.

Evans, D. (1961): Relapsed Club Foot. Journal of Bone and Joint Surgery, 43-B, 722.

Ghormley, R. K., and Allen, C. S. (1949): Progressive Deformities in Poliomyelitis. In Poliomyelitis. Papers and Discussions Presented at the First International Poliomyelitis Conference, p. 148. Philadelphia: J. B. Lippincott Company.

Green, W. T., and McDermott, L. J. (1942): Operative Treatment of Cerebral Palsy of Spastic Type. Journal of the American Medical Association, 118, 434.

Grice, D. S. (1952): An Extra-articular Arthrodesis of the Subastragalar Joint for Correction of Paralytic Flat Feet in Children. Journal of Bone and Joint Surgery, 34-A, 927.

Jones, R. (1911): Certain Operative Procedures in the Paralysis of Children with Special Reference to Poliomyelitis. British Medical Journal, ii, 1520.

Lovett, R. W. (1922): The Diagnosis, Prognosis and Early Treatment of Poliomyelitis. Journal of the American Medical Association, 78, 1607.

Mathews, S. S., Jones, M. H., and Sperling, S. C. (1953): Hip Derangements Seen in Cerebral Palsied Children. American Journal of Physical Medicine, 32, 213.

Medical Research Council (1942): War Memorandum No. 7. Aids to the Investigation of Peripheral Nerve Injuries. London: H.M. Stationery Office.

Mortens, J., and Pilcher, M. F. (1956): Tendon Transplantation in the Prevention of Foot Deformities after Poliomyelitis in Children. Journal of Bone and Joint Surgery, 38-B, 633.

Ober, F. R. (1936): The Rôle of the Iliotibial Band and Fascia Lata as a Factor in the Causation of Low-Back Disabilities and Sciatica. Journal of Bone and Joint Surgery, 18, 105.

Phelps, W. M. (1957): Long-term Results of Orthopaedic Surgery in Cerebral Palsy. Journal of Bone and Joint Surgery, 39-A, 53.

Pollock, G. A. (1953): Lengthening of the Gastrocnemius Tendon in Cases of Spastic Equinus Deformity. Journal of Bone and Joint Surgery, 35-B, 148.

Pollock, G. A. (1962): Surgical Treatment of Cerebral Palsy. Journal of Bone and Joint Surgery, 44-B, 68. 
Pollock, G. A., and Sharrard, W. J. W. (1958): Orthopaedic Surgery in the Treatment of Cerebral Palsy. In Recent Advances in Cerebral Palsy, p. 286. Edited by R. S. Illingworth. London: J. \& A. Churchill Ltd. Seddon, H. J. (1954): Poliomyelitis. Part II. Treatment of Poliomyelitis. In British Surgical Practice, Surgical Progress 1954, p. 162. London: Butterworth \& Co. (Publishers) Ltd.

Sharrard, W. J. W. (1955): Muscle Recovery in Poliomyelitis. Journal of Bone and Joint Surgery, 37-B, 63.

Sharrard, W. J. W. (1962): The Mechanism of Paralytic Deformity in Spina Bifida. Developmental Medicine and Child Neurology, 4, 310.

Sharrard, W. J. W. (1964a): The Nature and Management of Spasticity. The Peripheral Surgery of Spasticity. Proceedings of the Royal Society of Medicine, 57, 724.

Sharrard, W. J. W. (1964b): Posterior Iliopsoas Transplantation in the Treatment of Paralytic Dislocation of the Hip. Journal of Bone and Joint Surgery, 46-B, 426.

Stoffel, A. (1913): The Treatment of Spastic Contractures. American Journal of Orthopedic Surgery, 10, 611.

Strayer, L. M. (1950): Recession of the Gastrocnemius. An Operation to Relieve Spastic Contracture of the Calf Muscles. Journal of Bone and Joint Surgery, 32-A, 671.

Wiley, A. M. (1959): Club Foot. An Anatomical and Experimental Study of Muscle Growth. Journal of Bone and Joint Surgery, 41-B, 821.

Yount, C. C. (1926): The Rôle of the Tensor Fasciae Femoris in Certain Deformities of the Lower Extremities. Journal of Bone and Joint Surgery, 8, 171. 